\title{
Inner Thigh Rashes: A Complex And Polyedric Syndrome:A Solution Obtained By the Alternance of Two Natural Emulsions With Promised Remission in Seven Days.
}

\author{
Texia Research ${ }^{1}$, Lorenzo Martini $^{2}$ \\ ${ }^{1}$ Texia Innovazione Dermatologica-Turin, \\ ${ }^{2}$ Department Of Pharmaceutical Biotechnologies University Of Siena
}

\begin{abstract}
Inner Thigh Rashes (ITR) is a syndrome represented by mild skin lesions usually characterized as erythematous, slightly inflamed, and with either pruritus or burning sensation.It is typical in sportmen, joggers and bikers and depends on too many factors, as wetting, chafe and rubbing and perpetual contact with allergic fabric and their dyes during training. We have chosen a panel group of 11 bikers who like to ride $60 \mathrm{~km} /$ day and after having stated all the grades of the skin erythemas and all symptoms of ITR, we prayed them to apply two emulsions apt to cure all the causes that evoke ITR. To do this, we have prayed the volunteers to respond to questionnaires and we have examinated day after day for a week, noticing the eventual remission, that can be designated in 3-4 days for almost $70 \%$ of cases. We have treated a male, very pilous and presenting a pletora of blisters with an intense skin redness. This case was really particular, even if after one week some satisfying result has been reached.
\end{abstract}

Keywords: ITR, bikers, Zinc oxide, tocopherol, blisters.

\section{Background}

Inner Thigh Rashes (ITR) is a syndrome represented by mild skin lesions usually characterized as erythematous, slightly inflamed, and with either pruritus or burning sensation. This syndrome may be diagnosed in bikers and other sportmen, like joggers or motorcyclists who wear tigh pants made of cordura or gore-tex or kevlar and if neglected, this physio-pathological disorder can drive to important diseases, difficult to be cured.(1)

A variety of factors can cause this complicated syndrome.. These agents may be as simple as irritant, to as complex as an infection.

Amongst causes the following are the most important:

a) Contact Dermatitis: Rashes may occur whenever the skin comes in contact with an irritant. This goes for every part of the body, including the thighs.Contact dermatitis is a condition characterized by inflammation of the skin brought about by undesirable reactions to an irritant substance. These include bathing products, lotions, moisturizers, laundry detergents, and even the clothing itself, when made of synthetic fabric or an irritant dye.

b) Atopic Dermatitis or Eczema:One of the most common causes of eczema is atopic dermatitis. It is a chronic skin condition resulting from hypersensitivity of the skin. This usually leads to erythema, dryness, pruritus and swelling of the skin. In far more serious cases, this can occur as blisters, with either hyper or hypopigmentation of the skin. Once scratched, the areas may turn red and leather-like in character. Fabrics, especially of intime underwear, are the chief culprits. (2)

c) Excessive Heat and Sweat: Extreme heat and sweat can block sweat glands and their ducts. These eventually lead to inflammation, blister formation, along with appearance of itchy rashes. They are usually in the areas of skin folds and friction, such that on the inner thighs.Factors like humidity, clothing, physical activity, immature sweat ducts, and use of heavy creams and lotions can contribute to development of such rashes.

d) Activities Like Jogging and Cycling:Due to humidity, heat, sweat and friction, activities such as jogging and cycling may also lead to inner thigh rashes. When caused by cycling, these are usually coined as saddle sores. $(3,4)$

Either of these activities may lead to lesions characterized as chafed skin, which when neglected, may lead to intense blisters with pus formation known as folliculitis. For more severe cases, these may lead to large, swollen and painful abscesses.

e) Fungal Infections:Otherwise known as jock itch, one of the most common fungal skin infections is usually caused by tinea cruris. Manifestations of which include severe pruritus of skin folds of the thigh, along with erythematous rashes that usually blister and produce pus. The rashes are usually presented as sharply bordered, red patches and inguinal zones are always the most affected. 
The following indeed are the typical manifestations of a rash on the Inner Thigh Dermatoses and the usual signs and symptoms present on a patient with inner thigh skin lesions:

Pruritus

Erythema on the affected area

Blister formation $(5,6)$

Swelling and inflammation

Skin irritation

Pain and discomfort on ambulation

Here follow some common and traditional home made remedies to manage Inner Thigh Rashes:

Olive oil, thta can relieve skin tenderness and decrease its redness.

Ice application every few hours, and even cold water washes, can soothe uncomfortable skin lesions.

Ascorbic Acid supplements should be taken for better wound healing.

Lotion with aloe vera can be applied topical to further alleviate skin irritation.

Oatmeals can be used in bathing to relieve the inner thighs of their rashes.

Chamomile tea wash may diminish skin irritability.

Apple cider vinegar, mixed with honey and taken three times a day, may assist in controlling further skin allergies.

A concoction of cod liver and vitamin E cream may be applied on the rashes for a quicker cure.

Herbs, such as neem and licorice, can help rid of toxins, reducing rashes.

Here follow indeed some therapeutic modalities for Inner Thigh Rashes:

\section{Anti-Allergic Medications}

1) Antihistamines can diminish skin eruptions and diminish redness and irritation.

2) Corticosteroids

(These medications can decrease the inflammation and pruritic symptoms. These are mostly utilized for chronic eczematous rashes)

3) Moisturizing Products (The skin should always be well hydrated). This can be achieved with the use of moisturizing creams, mainly composed of retinoids, which are derivatives of Vitamin A, and vitamin D. They do not only provide hydration, but also maintain the skin's nourishment.

4) Immunosuppressants (These drugs can help diminish the severity of rashes, and at the same time, help the integument retain its normal color)

5) Phototherapy (As a skin therapy breakthrough, ultraviolet rays are found to be useful in decreasing the production of dermal cells. Thus, these assist in alleviating this skin nuisance).

\section{Here a short vademecum that intends to teach How to Prevent Inner Thigh Rashes:}

This skin manifestation is a huge part of the itch-scratch-itch cycle. Thus, it would be wise if this cycle is prevented. The following are ways of doing so:

1)After bathing, completely pat dry the thigh and perineal areas.

2)If one sees beginning manifestations of those rashes, he /she should wash the affected regions two times a day using cold water.

3)Clothes, loose-fitting and made of cotton, hemp or linen should be used. One must avoid frequent wearing of tight pants and undergarments.

4)Talcum powder can be used cool the thigh areas and avoid further skin irritation.

Modern technologies in manufacturing apposite fabric for bikers to lessen chafing have chose chamois. This soft cloth not only protected best against abrasion, it also provided wicking, meaning that it absorbs moisture from the skin, carrying it to the surface where air currents could dry it. Today's liners are contoured to fit the groin area, and they extend from front to back, cut in a Y- or an hourglass shape. The chamois, usually synthetic, will have several layers: the layer that comes in direct contact with the skin will be a soft "ultrasuede" fabric; the next layer, a cotton terry cloth with wicking properties; the third layer, a foam or gel cushion, and the outer-most layer, a close-knit fabric such as nylon or combination nylon/Lycra for extra protection against outside elements. Current versions of the chamois are also treated with an antibacterial agent to prevent fungal infections (similar to athlete's foot) in the groin area.

Chamois is very expensive and so the common fabric used in bicycle shorts usually consists of a blend of polyester, cotton, spandex, and nylon. Regardless of the materials used, they are usually spun and then combined into a single fabric. In spinning, filaments are drawn out of a spinneret, a device that works much like a shower head. Some filaments (such as spandex) are spun into an acid bath, while others are spun into open air. 
After combining the threads into a single fabric and then washing and dyeing, the fabric is cut into various panels.

Other fabric are polyamide (as nylon), polyurethane, (as Lycra), acrylic, merinos wool, especially for cold seasons.

In this research we have attempted to treat, case by case, the causes that can evoke the syndrome, contempling the diverse factors a, b, c, d and e, employing two cosmetic items that comprehend one or more ingredients apt to alleviate and cure the cause.

For example:

to combat cause a we have chosen bisabolol and panthenol

to combat cause $b$ we have chosen hyaluronic acid and sweet almond oil

to combat cause $\mathrm{c}$ we have chosen urea, hypericum perforatum oil, Zinc oxide

to combat cause d we have chosen phytosphingosine, ceramide 3 and 6 , cholesterol and glyceril oleate

to combat cause e we have chosen chloroxylenol and silver citrate.

All these ingredients are retrievable in two formulas we have suggested bikers we have recruited for experimentations and the two formulas had to been spread generously alternatively before and after training, keeping on any account how many times the bikers ride during the day or the night.

\section{Materials And Methods}

We have recruited 11 (A,B,C,D,E,F,G,H,I,L,M where Case C and G are the males and G the pilous one, Case E is albino) bikers who wear different types of pants made of diverse fabric and we think it is not necessary to list them, in this seat.

9 of those volunteers were female and 2 were male.

Of te males, the former was fully depilated in chest and inner thigh, the latter was completely pilous so that physiologically speaking could be defined "crinite" and showed red blisters on the inner thigh.

\section{The two creams we have employed to assure a complete remission contained the former the following ingredients:}

hypericum perforatum oil

calendula/jojoba/rice brain glyceryl 3-esters

squalane

tocopherol (6)

Zinc Oxide

The latter contained:

sweet almond oil

sodium hyaluronate

bisabolol

ceresin

silver citrate

phytosphingosine

ceramide 3 and 6 ,

cholesterol

glyceril oleate.

All the experimentations lasted one entire week.

The volunteers had to spread generously the former cream till complete absorption before riding, and had to apply the latter, after the training and after having a shower.

The volunteers had to respond to three questionnaires for seven days (in the morning at 6 o'clock before to ride 40-50 km).

\section{The questionnaires were based:}

1) on the presence or less of feeling of severe itching (Legend: bad: stands for pruritus, Ok stands for no feeling of pruritus)

2) Sensation of Discomfort on ambulation (Legend: bad: stands for discomfort, Ok stands for no difficulty of ambulating)

3) Skin Redness and blisters. (Table III will score both the number of blisters that day by day tend to disappear and the nuance of redness, that is measured by the Gallego-Sanz's scale.(8)

Here is the Gallego-Sanz's scale: 
Crimson

Lust

Terra cotta

Cerise

Rose.

\section{Results}

Here follow four tables (I,II,III and III $^{2}$ ) where all symptoms are observed day after day and plotted.

Table I: feeling of itching recorded during the seven days of treatment

\begin{tabular}{|l|l|l|l|l|l|l|l|}
\hline Case & $\begin{array}{l}\text { Itching at 1st } \\
\text { day }\end{array}$ & $\begin{array}{l}\text { Itching at 2nd } \\
\text { day }\end{array}$ & $\begin{array}{l}\text { Itching at } \\
\text { 3rd day }\end{array}$ & $\begin{array}{l}\text { Itching at } \\
\text { 4th day }\end{array}$ & $\begin{array}{l}\text { Itching at } \\
\text { 5th day }\end{array}$ & $\begin{array}{l}\text { Itching at } \\
\text { 6th day }\end{array}$ & $\begin{array}{l}\text { Itching at 7th } \\
\text { day }\end{array}$ \\
\hline A & bad & bad & bad & ok & ok & ok & ok \\
\hline B & bad & bad & ok & ok & ok & ok & ok \\
\hline C & bad & bad & bad & bad & ok & ok & ok \\
\hline D & bad & ok & ok & ok & ok & ok & ok \\
\hline E & bad & bad & bad & bad & ok & ok & ok \\
\hline F & bad & bad & ok & ok & ok & ok & ok \\
\hline G & bad & bad & bad & bad & bad & ok & ok \\
\hline H & bad & ok & ok & ok & ok & ok & ok \\
\hline I & bad & bad & bad & ok & ok & ok & ok \\
\hline L & bad & bad & ok & ok & ok & ok & ok \\
\hline M & bad & ok & ok & ok & ok & ok & ok \\
\hline
\end{tabular}

Table II: abilities in ambulating recorded during the seven days of treatment

\begin{tabular}{|l|l|l|l|l|l|l|l|}
\hline Case & & & & & & & \\
\hline A & bad & bad & bad & ok & ok & ok & ok \\
\hline B & bad & bad & ok & ok & ok & ok & ok \\
\hline C & bad & bad & bad & bad & ok & ok & ok \\
\hline D & bad & ok & ok & ok & ok & ok & ok \\
\hline E & bad & bad & bad & bad & ok & ok & ok \\
\hline F & bad & bad & ok & ok & ok & ok & ok \\
\hline G & bad & bad & bad & ok & ok & ok & ok \\
\hline H & bad & ok & ok & ok & ok & ok & ok \\
\hline I & bad & bad & bad & ok & ok & ok & ok \\
\hline L & bad & bad & ok & ok & ok & ok & ok \\
\hline M & bad & ok & ok & ok & ok & ok & ok \\
\hline
\end{tabular}

Table III: (1): Number of blisters disappearing day by day during the seven days of treatment:

\begin{tabular}{|l|l|l|l|l|l|l|l|}
\hline Case & 1st day & 2nd day & 3rd day & 4th day & 5 th day & 6th day & 7 th day \\
\hline A & 6 & 6 & 4 & 4 & 3 & 1 & 0 \\
\hline B & 5 & 5 & 5 & 4 & 2 & 0 & 0 \\
\hline C & 9 & 9 & 8 & 8 & 7 & 6 & 2 \\
\hline D & 7 & 7 & 4 & 3 & 3 & 2 & 0 \\
\hline E & 8 & 7 & 6 & 5 & 4 & 2 & 0 \\
\hline F & 6 & 6 & 5 & 3 & 2 & 1 & 0 \\
\hline G & 10 & 9 & 9 & 9 & 9 & 6 & 2 \\
\hline H & 5 & 5 & 4 & 3 & 1 & 1 & 0 \\
\hline I & 6 & 6 & 5 & 4 & 3 & 4 & 0 \\
\hline L & 9 & 9 & 8 & 7 & 6 & 2 & 2 \\
\hline M & 7 & 7 & 6 & 5 & 4 & & 0 \\
\hline
\end{tabular}

Table III(2): the nuance of redness observed day by day during the seve days of treatment

\begin{tabular}{|l|l|l|l|l|l|l|l|}
\hline Case & Redness & Redness & Redness & Redness & Redness & Redness & Redness \\
\hline A & Crimson & Crimson & Crimson & Terra cotta & Cerise & Cerise & Rose \\
\hline B & Crimson & Crimson & Crimson & Cerise & Rose & Rose & Rose \\
\hline C & Crimson & Crimson & Crimson & Crimson & $\begin{array}{l}\text { Terra } \\
\text { cotta }\end{array}$ & Terra cotta & Cerise \\
\hline D & Lust & Lust & Lust & Lust & Lust & Cerise & Cerise \\
\hline E & Lust & Cerise & Rose & Rose & Rose & Rose & Rose \\
\hline F & Lust & Lust & Lust & Lust & Lust & Lust & Cerise \\
\hline G & Crimson & Crimson & Crimson & Crimson & Crimson & Crimson & Terra cotta \\
\hline H & Terra cotta & Terra cotta & Terra cotta & Cerise & Cerise & Rose & Rose \\
\hline I & Lust & Lust & Lust & Lust & Lust & Lust & Cerise \\
\hline L & Crimson & Crimson & Lust & Lust & Cerise & Cerise & Rose \\
\hline M & Terra cotta & Terra cotta & Cerise & Cerise & Cerise & Rose & Rose \\
\hline
\end{tabular}




\section{Discussions and Conclusions}

It is mandatory to let the reader know that the pilous man (Case G) shows difficulties to get well.

Objectively it is well known that bikers generally depilate themselves.Effectively Case C, albeit mantains his nuance of redness (crimson) till 3rd day, shows a decrease of his blisters after 2 day. All the other cases manifest diverse typologies of remission, due to the phototype and Case E, especially, since she is an albino. Defintively, we can assure that using the two creams a restitutio ad integrum (or almost a decisive healing) is achieved after the 3rd-4th day in all cases, except Case G, even if after a week, blisters decrease from 10 to 2 .

\section{Aknowledgments:}

It has been possible carry out the experimentations and draft a paper thanks to two products called "Vitan Z" and "Flexian" produced by Texia srl in Turin.

\section{References}

[1]. Freedberg, et al:. Fitzpatrick's Dermatology in General Medicine. (6th ed.)2003; Ed. McGraw-Hill. p. 436

[2]. Berger W, Timothy J; Dirk E; . Andrews' Diseases of the Skin: Clinical Dermatology. (10th ed.). 2005.Ed Saunders. p. 125.

[3]. Beers MH, Porter RS, et al. The Merck Manual of Diagnosis and Therapy. 18th ed. Whitehouse Station, NJ: Merck Research Laboratories; 2006:1642-1644.

[4]. Passarini B, Infusino SD. Erythema nodosum. G Ital Dematol Venereol. 2013;148(4):413-7.

[5]. Requena L, Sanchez Yus E. Erythema Nodosum. Dermatologic Clinics. 2008;26(4).

[6]. Fuchs J, Kern H. Modulation of UV-light-induced skin inflammation by D-alpha-tocopherol and L-ascorbic acid: a clinical study using solar simulated radiation. Free Radic Biol Med. 1998;25(9):1006-1012.

[7]. Gallego R; Sanz, JC; Guía de coloraciones ; 2005.Ed. Gallego, Rosa; Sanz, Juan Carlos 